\title{
ESTUDO DA IMOBILIZAÇÃO DE LIPASE DE Rhizomucor miehei EM ORGANO-GEL PARA APLICAÇÃO EM SÍNTESE ORGÂNICA
}

\author{
K. F. CAVALCANTE ${ }^{1}$, F. A. PEREIRA ${ }^{2}$, L. R. B. GONÇALVES ${ }^{1}$, W. S. ADRIANO ${ }^{2}$ \\ ${ }^{1}$ Universidade Federal do Ceará, Departamento de Engenharia Química, Fortaleza-CE-Brasil \\ ${ }^{2}$ Universidade Federal de Campina Grande, Unidade Acadêmica de Saúde, Cuité-PB- Brasil \\ E-mail para contato: wellington.adriano@ufcg.edu.br
}

\begin{abstract}
RESUMO - Lipases, triacilglicerol éster hidrolases E.C. 3.1.1.3, são enzimas que atuam nas ligações ésteres de triacilgliceróis, liberando ácidos orgânicos e glicerol. Uma limitação da utilização destas enzimas reside na falta de estabilidade operacional e na impossibilidade de sua reutilização na forma livre. Visando contornar problemas como este, foram feitos estudos sobre imobilização de enzimas. Neste trabalho foram desenvolvidos derivados de lipases de Rhizomисor miehei imobilizadas em organo-géis, produzidos à base de gelatina (Gel), alginato (Alg) ou quitosana (Qui), fases orgânicas hexano (Hex) ou heptano (Hep) e os tensoativos dodecilsulfato de sódio (SDS) ou brometo de acetilmetilamônio (CTABr). Os derivados foram caracterizados quanto: fator de estabilidade a $60^{\circ} \mathrm{C}$, eficiência e rendimento de imobilização. Os melhores biocatalisadores apresentaram eficiência e fator de estabilidade de $4,1 \%$ e 30 vezes (Gel/SDS/Hex), 6,0\% e 1,3 vezes (Alg/SDS/Hep) e 1,0\% e 2,3 vezes (Qui/SDS/Hep).
\end{abstract}

\section{INTRODUÇÃO}

As enzimas atuam em condições suaves de temperatura, $\mathrm{pH}$ e pressão atingindo velocidades de reação bastante superior ao catalisadores químicos convencionais (KRAJEWSKA, 2004; HASAN, et al., 2006). Em sua forma solúvel, muitas enzimas não são suficientemente estáveis nas condições de reações desejáveis, devido a fatores como agitação mecânica, presença de solventes, altas temperaturas, $\mathrm{pH}$, necessidade de cofatores e sua inibição por altas concentrações de substrato e produtos, provocando a perda de sua atividade e especificidade. Visando contornar esse problema e promover a reutilização destas enzimas têm sido realizados estudos sobre imobilização de enzimas (VOLPATO, 2009).

Lipases (E.C. 3.1.1.3) são enzimas hidrolíticas que atuam catalisando a hidrólise de lipídeos para liberar ácidos orgânicos e glicerol (MENDES et al., 2013). Também são capazes de catalisar a reação reversa sob condições microaquosas, como por exemplo, a formação de ésteres a partir de álcoois e ácidos carboxílicos (YAHYA et al., 1998, PAIVA et al., 1997).

Recentemente tem-se investigado o potencial catalítico de lipases imobilizadas em organogéis, onde é obtido pela mistura de uma solução de polímero em água, solvente orgânico e tensoativo. Neste sistema a enzima está localizada no centro micelar (centro aquoso) do organogel, eliminando os problemas como de estabilização da enzima contra inativação por um solvente 
não-aquoso. O sistema final é um gel homogêneo, cuja consistência e propriedades físicas dependem da concentração relativa do polímero e água (JESUS et al., 1997).

Neste contexto, o objetivo deste trabalho foi desenvolver e caracterizar derivados de lipases de Rhizomucor miehei imobilizadas em organo-géis.

\section{MATERIAIS E MÉTODOS}

\subsection{Materiais}

Como suportes para imobilização utilizou-se gelatina em pó incolor sem sabor, alginato de sódio P.A e quitosana em pó (grau de desacetilação de 85, 9\%). Para a preparação dos suportes, utilizaram-se os reagentes: hexano P.A, N- heptano P.A), dodecilsulfato de sódio (SDS), brometo de acetilmetilamônio (CTABR), ácido acético glacial 95\%, álcool etílico P.A, dentre outros reagentes todos de grau analítico e de diversas marcas. Como agente ativante utilizou-se o glutaraldeído $25 \%$ (v/v).

\subsection{Métodos}

Preparação e imobilização da enzima em organo-gel utilizando gelatina em pó: A metodologia utilizada foi adaptada de DALLA-VECCHIA et al., (2004). Adicionou-se $100 \mu \mathrm{L}$ de enzima em $1 \mathrm{~mL}$ de água destilada e $0,2 \mathrm{~g}$ de gelatina, a mistura foi realizada a $50^{\circ} \mathrm{C}$. Dissolveu-se $0,04 \mathrm{~g}$ do tensoativo em $4 \mathrm{~mL}$ da fase orgânica. Os sistemas foram agitados por 10 minutos e armazenados em geladeira por 5 minutos.

Preparação do organo-gel utilizando gelatina seguido de ativação com glutaraldeído $2 \%$ (v/v): O preparo do organo-gel foi de acordo com o descrito anteriormente, sem adição da enzima. Em seguida ativados com glutaraldeído $2 \%$ (v/v) em tampão fosfato $20 \mathrm{mM}, \mathrm{pH} 8,0$, sob agitação a $25^{\circ} \mathrm{C}$ por 10 minutos. $\mathrm{O}$ suporte foi lavado com água destilada para remoção do glutaraldeído residual e filtrado a vácuo.

Preparação e imobilização da enzima em organo-gel utilizando alginato de sódio: $0,04 \mathrm{~g}$ de alginato de sódio e $2 \mathrm{~mL}$ de água destilada foram misturados por 10 minutos. Mediu-se $0,04 \mathrm{~g}$ do tensoativo (SDS), $4 \mathrm{~mL}$ da fase orgânica e adicionou-se $180 \mu \mathrm{L}$ da enzima. Em seguida, o organogel foi submerso em solução de $\mathrm{CaCl}_{2} 5 \%(\mathrm{~m} / \mathrm{v})$ por 5 minutos, para que ocorresse a coagulação do alginato e formação de partículas. Lavou-se o organo-gel com tampão fosfato de sódio $25 \mathrm{mM}$, pH 7,0, com a fase orgânica e filtrado a vácuo.

Preparação do organo-gel utilizando alginato de sódio seguido de ativação com glutaraldeído $2 \%(\mathrm{v} / \mathrm{v})$ : O organo-gel foi preparado de acordo com o descrito no item anterior, sem adição da enzima. Foi realizado o processo de imobilização nos suportes produzidos. Os suportes foram lavados com água destilada e secos em bomba a vácuo e suspensos em soluções de glutaraldeído $2 \%(\mathrm{v} / \mathrm{v})$ por 10 minutos. O suporte foi lavado com água destilada e filtrado a vácuo.

Preparação e imobilização da enzima em organo-gel utilizando quitosana em pó: Preparouse uma solução de ácido acético $5 \%$ (v/v), pH ajustado para 5,0 e adicionou-se quitosana até 
concentração de $5 \%(\mathrm{~m} / \mathrm{v})$. Mediu-se $0,04 \mathrm{~g}$ do tensoativo SDS, $1 \mathrm{~mL}$ da fase orgânica, adicionouse a enzima e $4 \mathrm{~g}$ da solução de quitosana. O organo-gel foi colocado em geladeira por 20 minutos, decorrido o tempo adicionou-se tampão bicarbonato de sódio $25 \mathrm{mM}, \mathrm{pH} 10,0$ por 30 minutos, para que ocorresse a neutralização do $\mathrm{pH}$ e coagulação da quitosana, em seguida adicionou-se em álcool etílico $99,5 \%$ por 10 minutos, até ficar com consistência de gel, em seguida lavado com o solvente e filtrado a vácuo.

Processo de imobilização da enzima ao suporte: A imobilização da lipase nos suportes ativados com glutaraldeído $2 \%(\mathrm{v} / \mathrm{v})$ foi feita através do contato desses suportes com uma solução de enzima, carga oferecida $50 \mathrm{U} / \mathrm{g}$ de gel, dissolvida em tampão fosfato de sódio $20 \mathrm{mM}$, pH 8, na razão de $1 / 10(\mathrm{~m} / \mathrm{v})$, incubados a $25^{\circ} \mathrm{C}$ por 3horas. Decorrido tempo, os derivados foram lavados com água destilada e secos a vácuo.

Determinação da atividade hidrolítica da enzima solúvel e imobilizada: A atividade hidrolítica da enzima solúvel e imobilizada foi determinada pela hidrólise do pNPB, avaliada espectrofotometricamente com comprimento de onda $400 \mathrm{~nm}$, de acordo com a Equação 1 (OLIVEIRA, 2012).

$$
A t=\frac{\left(\alpha-\alpha_{\text {branco }}\right) \times 0,0922 \times V_{\text {reator }}(\mathrm{mL})}{V_{\text {enzima }}(\mathrm{mL}) \text { ou } m_{\text {derivado }}(g)}
$$

Estabilidade térmica: Para a avaliação da estabilidade térmica da lipase na forma solúvel, adicionou-se $0,2 \mathrm{~mL}$ da enzima em $2 \mathrm{~mL}$ tampão fosfato de sódio $25 \mathrm{mM}, \mathrm{pH} 7,0$ à $60^{\circ} \mathrm{C}$. As amostras foram retiradas em tempos estabelecidos e colocados em banho de gelo para interromper o processo de inativação e em seguida suas atividades foram determinadas. Para os ensaios feitos com a enzima imobilizada, mediu-se $0,1 \mathrm{~g}$ do derivado incubado em $1 \mathrm{~mL}$ da fase orgânica e incubados à $60^{\circ} \mathrm{C}$, retirados e colocados em banho de gelo. Em seguida, foi seco em bomba a vácuo e medida a atividade.

Parâmetros de inativação térmica e parâmetros de imobilização: Foi utilizado o ajuste exponencial não linear proposto por SADANA-HENLEY (1982) de acordo com a Equação 2, em seguida calculado a atividade relativa, tempo de meia-vida e fator de estabilidade. Para todos os derivados determinou-se a eficiência de acordo com a Equação 3 e para os derivados ativados com glutaraldeído $2 \%$ (v/v), determinou-se o rendimento de imobilização conforme Equação 4.

$$
\begin{aligned}
& A_{r}=(1-\alpha) x e^{-k d x t}+\alpha \\
& E(\%)=\frac{A t_{\text {derivado }}}{\text { Carga Oferecida }} \times 100 \\
& R I(\%)=\frac{\left(A t_{o}-A t_{f}\right)}{A t_{\circ}} \times 100
\end{aligned}
$$




\section{RESULTADOS E DISCUSSÃO}

Inicialmente foi medida a atividade hidrolítica da lipase de Rhizomucor miehei, apresentando um valor de 250,5 $\pm 1,9 \mathrm{U}_{\mathrm{PNPB}} \cdot \mathrm{mL}^{-1}$ de extrato enzimático $(6,87 \pm 0,01 \mathrm{mg}$ de Proteina. $\mathrm{mL}^{-1}$ de extrato enzimático ).

\subsection{Caracterização Da Lipase De Rhizomucor Miehei Imobilizada Em Organo- Géis À Base De Gelatina}

Os derivados preparados a partir da gelatina em pó apresentaram resultados de acordo com a Tabela 1. Observa-se que o melhor derivado obtido foi usando SDS como tensoativo e hexano como fase orgânica visto que o valor de eficiência foi de 4,1\%, com fator de estabilidade de 30 vezes.

Tabela 1-Caracterização de lipases imobilizadas em organo-géis à base de gelatina, utilizando hexano ou heptano como fases orgânicas e os tensoativos SDS ou CTABr. Sendo (At $t_{\text {Der }}$ ) Atividade do derivado, (E) Eficiência, (FE) Fator de estabilidade e ( $\mathrm{t}$ 1/2) Tempo de meia-vida

\begin{tabular}{|l|c|c|c|c|c|}
\hline \multicolumn{1}{|c|}{ Derivados } & $\begin{array}{c}\text { Carga Oferecida } \\
(\mathbf{U} / \mathbf{g})\end{array}$ & $\mathbf{E ~ ( \% )}$ & $\begin{array}{c}\mathbf{A t}_{\text {Der }} \\
\left(\mathbf{U}_{\mathbf{P N P B}} / \mathbf{g}\right)\end{array}$ & $\begin{array}{c}\mathbf{t}_{1 / 2} \\
(\mathbf{m i n})\end{array}$ & $\mathbf{F E}$ \\
\hline Enzima solúvel & - & - & - & 0,4 & 1,0 \\
\hline Gel/SDS/Hex & $37,8 \pm 1,4$ & $4,1 \pm 1,9$ & $1,4 \pm 0,3$ & $2,7 \pm 0,9$ & $30 \pm 2,6$ \\
\hline Gel/CTABr/Hex & $38,1 \pm 4,7$ & $0,7 \pm 0,6$ & $0,3 \pm 0,2$ & nd & nd \\
\hline Gel/SDS/Hep & $53,0 \pm 1,3$ & $2,7 \pm 0,9$ & $1,5 \pm 0,8$ & $13,1 \pm 8,4$ & $31,2 \pm 2,0$ \\
\hline Gel/CTABr/Hep & $61,2 \pm 2,4$ & $0,5 \pm 0$ & $0,3 \pm 0,1$ & nd & nd \\
\hline
\end{tabular}

*nd: não determinado

\subsection{Caracterização Da Lipase De Rhizomucor Miehei Imobilizada Em Organo- Géis À Base De Gelatina Ativados Com Glutaraldeído 2\% (V/V)}

De acordo com a Tabela 2, Observa-se que o derivado que apresentou melhores resultados foi Gelatina/SDS/Heptano/Glutaraldeído devido ao fator de estabilidade de 68,3 vezes mais estável que a enzima solúvel, apresentando tempo de meia-vida e rendimento de 28,6 minutos e $71,2 \%$, respectivamente. 
Tabela 2-Caracterização de lipases imobilizadas em organo-géis a base de gelatina, utilizando hexano ou heptano como fases orgânicas e os tensoativo SDS ou CTABr, ativados com glutaraldeído $2 \%(\mathrm{v} / \mathrm{v})$, incubados por 3horas sob agitação e a temperatura ambiente. Carga oferecida 50 U.g-1 de suporte. Sendo (At $t_{\text {Der }}$ ) Atividade do derivado, (E) Eficiência, (FE) Fator de estabilidade e ( $\mathrm{t}$ 1/2) Tempo de meia-vida, (RI) Rendimento de imobilização

\begin{tabular}{|c|c|c|c|c|c|}
\hline Derivados & $\mathbf{E ~ ( \% )}$ & $\begin{array}{c}\mathbf{A t}_{\text {Der }} \\
\left(\mathbf{U}_{\mathbf{P N P B}} / \mathbf{g}\right)\end{array}$ & $\begin{array}{c}\mathbf{t}_{1 / 2} \\
(\mathbf{m i n})\end{array}$ & $\mathbf{F E}$ & $\begin{array}{c}\text { RI } \\
(\mathbf{\%})\end{array}$ \\
\hline Enzima solúvel & - & - & 0,4 & 1,0 & - \\
\hline Gel/SDS/Hex/Glu & $1,1 \pm 0,1$ & $0,5 \pm 0,07$ & $7,5 \pm 0,4$ & $17,8 \pm 0,9$ & $77,0 \pm 2,4$ \\
\hline Gel/CTABr/Hex/Glu & $0,1 \pm 0$ & $0,06 \pm 0$ & nd & nd & nd \\
\hline Gel/SDS/Hep/Glu & $0,8 \pm 0,1$ & $0,5 \pm 0,07$ & $28,6 \pm 1,1$ & $68,3 \pm 2,8$ & $71,2 \pm 6,2$ \\
\hline Gel/CTABr/Hep/Glu & $0,06 \pm 0$ & $0,03 \pm 0$ & nd & nd & nd \\
\hline
\end{tabular}

*nd: não determinado

\subsection{Caracterização Da Lipase De Rhizomucor Miehei Imobilizada Em Organo- Géis À Base De Alginato De Sódio}

Observando-se a Tabela 3, verifica-se que os derivados produzidos obtiveram resultados bem próximos, o derivado Alginato/SDS/Heptano destaca-se por apresentar uma maior eficiência que o Alginato/SDS/Hexano, 6,0\% e 4,3\%, respectivamente. Observa-se que os dois derivados obtiveram valores de fator de estabilidade de aproximadamente 1,3 vezes.

Tabela 3-Caracterização de lipases imobilizadas em organo-géis à base de alginato de sódio, utilizando hexano ou heptano como fases orgânicas e o tensoativo SDS. Sendo (At $t_{\text {Der }}$ ) Atividade do derivado, (E) Eficiência, (FE) Fator de estabilidade e (t 1 1 ²) Tempo de meia-vida

\begin{tabular}{|l|c|c|c|c|c|}
\hline Derivados & $\begin{array}{c}\text { Carga Oferecida } \\
(\mathbf{U} / \mathbf{g})\end{array}$ & $\mathbf{E ~ ( \% )}$ & $\begin{array}{c}\mathbf{A t}_{\text {Der }} \\
\left(\mathbf{U}_{\mathbf{P N P B}} / \mathbf{g}\right)\end{array}$ & $\begin{array}{c}\mathbf{t}_{1 / 2} \\
(\mathbf{m i n})\end{array}$ & FE \\
\hline Enzima solúvel & - & - & - & 0,4 & 1,0 \\
\hline Alg/SDS/Hex & $18,2 \pm 0,8$ & $4,3 \pm 0,7$ & $0,8 \pm 0,1$ & $0,5 \pm 0,2$ & $1,3 \pm 0,7$ \\
\hline Alg/SDS/Hep & $14,1 \pm 0,6$ & $6,0 \pm 1,0$ & $0,8 \pm 0,1$ & $0,5 \pm 0,1$ & $1,3 \pm 0,2$ \\
\hline
\end{tabular}

\subsection{Caracterização Da Lipase De Rhizomucor Miehei Imobilizada Em Organo- Géis À Base De Alginato De Sódio Ativados Com Glutaraldeído 2\% (V/V)}

A Tabela 4 mostra que o derivado Alginato/SDS/Hexano/Glutaraldeído apresentou valores de rendimento e eficiência $82,6 \%$ e $0,5 \% \mathrm{~m} / \mathrm{v}$, respectivamente. Já Alginato/SDS/Heptano/Glutaraldeído apresentaram valores de rendimento 60,6 \% e eficiência 1,0 $\%$. 
Tabela 4-Caracterização de lipases imobilizadas em organo-géis à base de alginato de sódio, utilizando hexano ou heptano como fases orgânicas e o tensoativo SDS, ativados com

glutaraldeído $2 \%$ (v/v), incubados por 3 horas sob agitação e a temperatura ambiente. Carga oferecida 50 U.g-1 de suporte. Sendo (At $t_{\text {Der }}$ ) Atividade do derivado, (E) Eficiência, (FE) Fator de estabilidade e ( $\mathrm{t}$ 1/2) Tempo de meia-vida, (RI) Rendimento de imobilização

\begin{tabular}{|l|c|c|c|c|c|}
\hline \multicolumn{1}{|c|}{ Derivados } & E $(\boldsymbol{\%})$ & $\begin{array}{c}\mathbf{A t}_{\text {Der }} \\
\left(\mathbf{U}_{\mathbf{P N B}} / \mathbf{g}\right)\end{array}$ & $\begin{array}{c}\mathbf{t}_{1 / 2} \\
(\mathbf{m i n})\end{array}$ & FE & RI (\%) \\
\hline Enzima solúvel & - & - & 0,4 & 1,0 & - \\
\hline Alg/SDS/Hex/Glu & $0,5 \pm 0,1$ & $0,2 \pm 0,06$ & $0,1 \pm 0,05$ & $0,3 \pm 0,1$ & $82,6 \pm 5,5$ \\
\hline Alg/SDS/Hep/Glu & $1,0 \pm 0,3$ & $0,5 \pm 0,1$ & $0,1 \pm 0,02$ & $0,4 \pm 0,04$ & $60,6 \pm 1,9$ \\
\hline
\end{tabular}

\subsection{Caracterização Da Lipase De Rhizomucor Miehei Imobilizada Em Organo- Géis À Base De Quitosana}

Pode-se observar, de acordo com a Tabela 5, que os derivados à base de quitosana obtiveram resultados bem próximos, tendo maior destaque o derivado Quitosana/SDS/Heptano, pois apresentou melhores resultados, quando comparado com o derivado Quitosana/SDS/Hexano, de fator de estabilidade e tempo de meia-vida de 2,3 e 1,0 minutos, respectivamente. As eficiências de ambos os derivados apresentaram valores em torno de $1,0 \%$.

Tabela 5-Caracterização de lipases imobilizadas em organo-géis à base de quitosana, utilizando

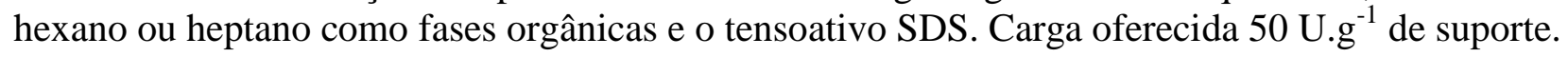
Sendo $\left(\mathrm{At}_{\text {Der }}\right)$ Atividade do derivado, $(\mathrm{E})$ Eficiência, $(\mathrm{FE})$ Fator de estabilidade e $(\mathrm{t} 1 / 2)$ Tempo de meia-vida

\begin{tabular}{|l|c|c|c|c|}
\hline \multicolumn{1}{|c|}{ Derivados } & $\mathbf{E ~ ( \% )}$ & $\begin{array}{c}\text { At } \\
\left(\mathbf{U}_{\mathbf{P N P B}} / \mathbf{g}\right)\end{array}$ & $\begin{array}{c}\mathbf{t}_{1 / 2} \\
(\mathbf{m i n})\end{array}$ & $\mathbf{F E}$ \\
\hline Enzima solúvel & - & - & 0,4 & 1,0 \\
\hline Qui/SDS/Hex & $1,0 \pm 0,1$ & $0,5 \pm 0,05$ & $0,8 \pm 0,08$ & $1,8 \pm 0,2$ \\
\hline Qui/SDS/Hep & $1,0 \pm 0,07$ & $0,5 \pm 0,03$ & $1,0 \pm 0,3$ & $2,3 \pm 0,6$ \\
\hline
\end{tabular}

\subsection{Determinação Dos Melhores Biocatalisadores}

Com base nos estudos feitos com os derivados produzidos a partir dos polímeros gelatina, alginato e quitosana, selecionaram-se os melhores biocatalisadores, Gelatina/SDS/Hexano, Alginato/SDS/Heptano, Quitosana/SDS/Heptano, de acordo com os resultados apresentados na Tabela 6. Foram selecionados os biocatalisadores produzidos pelo método de microencapsulação, onde a enzima encontra-se confinada dentro do centro micelar dos organo-géis. 
Tabela 6-Seleção dos melhores biocatalisadores. Sendo (E) Eficiência, (t 1⁄2) tempo de meia-vida e (FE) Fator de Estabilidade

\begin{tabular}{|c|c|c|c|}
\hline Derivados & $\mathbf{E}(\boldsymbol{\%})$ & $\begin{array}{c}\mathbf{t}_{1 / 2} \\
(\mathbf{m i n})\end{array}$ & $\mathbf{F E}$ \\
\hline Gel/SDS/Hex & $4,1 \pm 1,9$ & $12,7 \pm 0,9$ & $30,0 \pm 2,6$ \\
\hline Alg/SDS/Hep & $6,0 \pm 1,0$ & $0,5 \pm 0,1$ & $1,3 \pm 0,2$ \\
\hline Qui/SDS/Hep & $1,0 \pm 0,07$ & $1,0 \pm 0,3$ & $2,3 \pm 0,6$ \\
\hline
\end{tabular}

A Figura 1 mostra os perfis de inativação térmica a $60{ }^{\circ} \mathrm{C}$ dos melhores biocatalisadores preparados através da imobilização em suportes à base de gelatina, alginato e quitosana. As linhas indicam ajuste do modelo de decaimento exponencial de Sadana-Henley aos pontos experimentais.

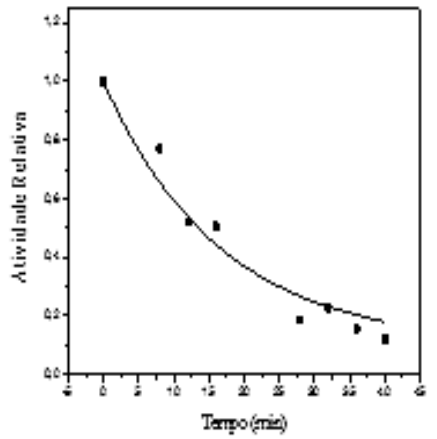

(a)

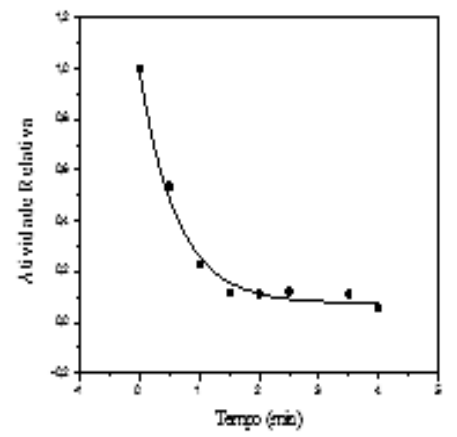

(b)

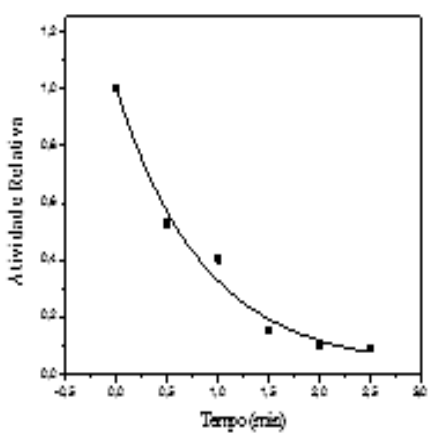

(c)

Figura 1- Perfil de inativação térmica a $60{ }^{\circ} \mathrm{C}$ da lipase de Rhizomucor miehei imobilizada em (a) Gelatina/SDS/Hexano, (b) Alginato/SDS/Heptano e (c) Quitosana/SDS/Heptano.

\section{CONCLUSÃO}

Foi avaliada a estabilidade térmica como uma estratégia para escolha dos melhores derivados, observou-se que os derivados que obtiveram melhor desempenho foram Gel/SDS/Hex que apresentou eficiência de 4,1\%, tempo de meia-vida de 12,7 minutos e 30 vezes mais estável que a enzima na sua forma livre, Alg/SDS/Hep apresentou eficiência 6,0\%, tempo de meia-vida 0,5 minutos e 1,3 vezes mais estável, Qui/SDS/Hep apresentou eficiência 1,0\%, tempo de meiavida $1,0 \%$ e 2,3 vezes mais estável.

A metodologia de imobilização seguida de ativação por glutaraldeído $2 \%(\mathrm{v} / \mathrm{v})$, apesar de apresentar bons resultados de rendimento, tempo de meia-vida e fator de estabilidade, não apresentou boa atividade do derivado e eficiência causada pela redução dos poros após reticulação, dificultando $\mathrm{o}$ acesso do substrato ao sitio ativo. $\mathrm{O}$ uso do brometo de 
hexadeciltrimetilamônio (CTABr) não apresentou bons resultados, devido inativação da enzima causado pelo tensoativo.

\section{REFERÊNCIAS}

DALLA-VECCHIA, R.; NASCIMENTO, M. G.; SOLDI, V. Aplicações cinéticas de lipases imobilizadas em polímeros, Química Nova, v. 27, p. 623-630, 2004.

HASAN, F.; SHAH, A.A., HAMEED, A. Industrial applications of microbial lipases. Enzyme and Microbial Technology; v. 39; p. 235, 2006.

JESUS, P.C.; JOÃO, J.J.; SILVA, P.L.F.; BURLIN, G.; NASCIMENTO, M.G. Organo-gel: um novo sistema para a imobilização de lipases e sua aplicação em síntese orgânica. Química Nova, v. 20, p. 664-672, 1997.

KRAJEWSKA, B. Application of chitin- and chitosan-based materials for enzyme immobilizations: A review. Enzyme and Microbial Technology, v.35, p.126-139. 2004.

MENDES, A. A.; CASTRO, H.F.; GIORDANO, R. L.C. Triagem de Suportes Orgânicos e Protocolos de Ativação na Imobilização e Estabilização de Lipase de Thermomyces Lanuginosus. Quimica Nova, v. 36, n . 2, p. 245-251, 2013.

OLIVEIRA, U.M.F. Síntese de Ésteres de Interesse Comercial Utilizando Lipases Imobilizadas em Quitosana. 2012. 191 f. Tese (Doutorado em Biotecnologia)- Rede Nordeste de Biotecnologia- RENORBIO, Universidade Estadual do Ceará, Fortaleza, 2012.

PAIVA, A. L; MALCATA, F. X. Integration of reaction and separation with lipases: An overview. Journal of Moecular Catalysis B: Enzymatic,v. 3, p. 99-109, 1997.

SADANA, A., HENLEY, J.P. Analysis of enzyme deactivations by a series-type mechanism:influence of modification on the activity and stability of enzymes. Ann N Y Acad Sci, v. 501, p.73-79, 1987.

VOLPATO, G. Produção, Purificação e Imobilização de Lipases de Staphylococcus warneri EX17 Produzidas em Glicerol. 2009. 147 f. Tese (Doutorado em Engenharia Quimica), Universidade Federal do Rio Grande do Sul, Rio Grande do Sul, 2009.

YAHYA, A. R. M.; ANDERSON, W. A.; MOO-YOUNG, M. Ester synthesis in lipase catalyzed reactions. Enzyme Microbiology Technology, v.23, p. 438-450, 1998. 